\title{
Stability of titania nanoparticles in soil suspensions and transport in saturated homogeneous soil columns
}

\author{
Jing Fang ${ }^{a}$, Xiao-quan Shan ${ }^{\mathrm{a}, *}$, Bei Wen ${ }^{\mathrm{a}, *}$, Jin-ming Lin ${ }^{\mathrm{a}}$, Gary Owens ${ }^{\mathrm{b}}$ \\ a State Key Laboratory of Environmental Chemistry and Ecotoxicology, Research Center for Eco-Environmental Sciences, Chinese Academy of Sciences, P.O. Box 2871, \\ Beijing 100085, China \\ ${ }^{\mathrm{b}}$ Centre for Environmental Risk Assessment and Remediation, University of South Australia, Mawson Lakes, SA 5095, Australia
}

$\mathrm{TiO}_{2}$ nanoparticles could efficiently suspend in soil suspensions and potentially transport to deeper soil layers.

\section{A R T I C L E I N F O}

Article history:

Received 19 May 2008

Received in revised form

30 October 2008

Accepted 5 November 2008

\section{Keywords:}

Titanium dioxide

Stability

Soil suspension

Transport

Soil columns

\begin{abstract}
A B S T R A C T
The stability of $\mathrm{TiO}_{2}$ nanoparticles in soil suspensions and their transport behavior through saturated homogeneous soil columns were studied. The results showed that $\mathrm{TiO}_{2}$ could remain suspended in soil suspensions even after settling for 10 days. The suspended $\mathrm{TiO}_{2}$ contents in soil suspensions after $24 \mathrm{~h}$ were positively correlated with the dissolved organic carbon and clay content of the soils, but were negatively correlated with ionic strength, $\mathrm{pH}$ and zeta potential. In soils containing soil particles of relatively large diameters and lower solution ionic strengths, a significant portion of the $\mathrm{TiO}_{2}(18.8-$ $83.0 \%$ ) readily passed through the soils columns, while $\mathrm{TiO}_{2}$ was significantly retained by soils with higher clay contents and salinity. $\mathrm{TiO}_{2}$ aggregate sizes in the column outflow significantly increased after passing through the soil columns. The estimated transport distances of $\mathrm{TiO}_{2}$ in some soils ranged from 41.3 to $370 \mathrm{~cm}$, indicating potential environmental risk of $\mathrm{TiO}_{2}$ nanoparticles to deep soil layers.
\end{abstract}

(c) 2008 Elsevier Ltd. All rights reserved.

\section{Introduction}

$\mathrm{TiO}_{2}$ nanoparticles are commonly used in cosmetics, sunscreens, paints, coatings, and increasingly for the photocatalytic degradation of various pollutants in water, air and soil matrices (Higarashi and Jardim, 2002; Nagaveni et al., 2004; Quan et al., 2005; Aarthi and Madras, 2007) and have also been used to successfully immobilize anions in soils (Mattigod et al., 2005). However, $\mathrm{TiO}_{2}$ nanoparticles may also pose potential risks to human health and ecosystems when applied in commercial quantities (Wiesner et al., 2006). Therefore, it is essential to understand the fate and transport of $\mathrm{TiO}_{2}$ nanoparticles in the environment in order to assess possible routes of exposure to humans and the ecosystem.

At the nano-scale, attractive dispersion interactions, known as London-van der Waals forces, start to become important and often equilibrate with the inertia of the particles (Hamaker, 1937) such that although many nanomaterials are produced with a small targeted size, nanoparticles frequently form much larger colloidal aggregates (Wiesner et al., 2006). Only a few reports on the stability of $\mathrm{TiO}_{2}$ nanoparticles in aqueous solution have appeared in the

\footnotetext{
* Corresponding authors. Tel.: +8610 62923560; fax: +86 1062923563. Wen).
}

literature. Anatase particles $(20 \mathrm{~nm})$ being one of the three mineral forms of titanium dioxide, had a tendency to form aggregates with a narrow size distribution of $\sim 200 \mathrm{~nm}$ size which were stable in pure water (Wiesner et al., 2006). Solution pH and surface charge mainly govern the stability of $\mathrm{TiO}_{2}$ nanoparticles in aqueous solution (Barringer and Bowen, 1985; Kallay and Zalac, 2002; Guzman et al., 2006). Guzman et al. (2006) reported that over $80 \%$ of suspended $\mathrm{TiO}_{2}$ nanoparticles were mobile in microchannels over the $\mathrm{pH}$ range of $1-12$, except where the $\mathrm{pH}$ was close to the zero point change for $\mathrm{TiO}_{2}\left(\mathrm{pH}_{\mathrm{pzc}}=6.2\right)$. Inorganic salts also had direct effects on nanoparticle aggregation (Snoswell et al., 2005) and additives such as surfactants and organic solvents significantly improved the dispersion of $\mathrm{TiO}_{2}$ nanoparticles in aqueous solutions (Hsu and Chang, 2000; Tkachenko et al., 2006). In the natural environment, groundwater aquifers and surface waters typically have high concentrations of calcium or magnesium, which would favor aggregation and deposition of nanoparticles (Wiesner et al., 2006). While natural organic matter (NOM) is expected to have important influences on most physicochemical reactions, the exact role of NOM in colloid stabilization or destabilization would primarily depend on the chemical properties such as size, charge, and rigidity of various functional groups (Wilkinson et al., 1997). Aquagenic biopolymers could increase the coagulation rate of colloidal montmorillonite, while fulvic acids were shown to stabilize colloidal solutions (Wilkinson et al., 1997). Humic acid was also 
frequently reported for effectively stabilizing nanoparticle suspensions by steric repulsion effect (Chen and Elimelech, 2007; Hyung et al., 2007). In soil systems, where ionic strengths of the soil solutions are usually less than 0.005 M (Black and Campbell, 1982), fulvic acids and humic acids are important portions of the dissolved soil organic matter (Van Hees et al., 2005), and this would favor the stabilization of inorganic colloids indicating that $\mathrm{TiO}_{2}$ nanoparticle can potentially be well dispersed in soil solutions.

Dispersion of nanoparticles in the environment implies a higher mobility and a greater potential for risk of exposure since well dispersed nanoparticles will be transported longer distances, exhibit longer effective persistence, and be potentially involved in particle-facilitated contaminant movement (Ryan and Elimelech, 1996; Sen and Khilar, 2006; Zhuang et al., 2003). Therefore, knowledge of the transport of $\mathrm{TiO}_{2}$ nanoparticles in soil media is important for developing an understanding of the potential mobility of nanoparticles in general in the soil environment and their potential risks to groundwater. The transport of colloids and nanoparticles in porous media is impeded by two processes, 1) straining or physical filtration where the particle is larger than the pore and is trapped and 2) true filtration where the particle is removed from solution by interception, diffusion and sedimentation (Nowack and Bucheli, 2007). Recently, a few investigations have addressed the transport of nanoparticles in porous media (Guzman et al., 2006; Lecoanet et al., 2004; Lecoanet and Wiesner, 2004) and showed that nanoparticles including carbon nanomaterials, anatase and silica exhibited various transport behaviors. The passage of anatase through a two-dimensional model of porous structure column slowly increased with time, suggesting that the mobility was increased with time as deposition sites became saturated (Guzman et al., 2006). Darcy velocities could also influence transport and deposition of nanoparticles and an increase in the flow velocity increased the effluent of anatase (Lecoanet et al., 2004). However, most of these experiments were conducted in well-defined porous media, glass or polystyrene bead-packed columns and thus did not accurately represent the variety of mineral surface types, surface charge heterogeneities, grain size distributions and roughness of granular media encountered in real soil systems. Thus the environmental implications of such studies involving well-defined porous media were limited.

Soils are complicated assemblies of solid, liquids, and gases (Reilly, 2002). Soil properties such as size, surface area, charge behavior, types of minerals and organic matters will greatly affect the aggregation and transport of nanoparticles (Kretzschmar et al., 1997). Hence, the objectives of this study were to investigate the degree to which $\mathrm{TiO}_{2}$ nanoparticles were suspended in various soil suspensions and to investigate how far $\mathrm{TiO}_{2}$ nanoparticles could be transported in various homogeneous soil columns.

\section{Theory}

Particle transport in porous media can be described by three mechanisms: 1) direct interaction of the particles with the media, 2 ) sedimentation due to gravity and 3) diffusion due to Brownian motion (Yao et al., 1971; Guzman et al., 2006). For nanoparticles, since gravitational sedimentation is negligible (Tufenkji and Elimelech, 2004) only two mechanisms are significant. Under steady saturated flow conditions the transport of particles through porous media can be described by a convective-dispersive transport equation including a term for first-order particle deposition (Kretzschmar et al., 1997):

$\frac{\partial C}{\partial t}=D \frac{\partial^{2} C}{\partial x^{2}}-v_{\mathrm{p}} \frac{\partial C}{\partial x}-k C$

where $C$ is the particle concentration in solution, $t$ is the elapsed time, $x$ is the travel distance, $D$ is the dispersion coefficient for particles, $\nu_{\mathrm{p}}$ is the average travel velocity of particles, and $k$ is the particle deposition rate coefficient.

Based on the assumption of first-order deposition kinetics, particle breakthrough curves resulting from step-inputs are often evaluated by calculating a clean-bed filter coefficient given by:

$\lambda_{0}=-\frac{1}{L} \ln \left(\frac{C_{\mathrm{f}}}{C_{0}}\right)$

where $L$ is the column length, $C_{0}$ is the initial effluent particle concentration, and $C_{\mathrm{f}}$ is the final effluent particle concentration after the breakthrough curve has reached a plateau. The particle deposition rate coefficient $k$ can then be estimated using:

$k=\lambda_{0} v_{\mathrm{p}}=\lambda_{0} L / t_{\mathrm{p}}$

and thus

$k=-\frac{1}{t_{\mathrm{p}}} \ln \left(\frac{C_{\mathrm{f}}}{C_{0}}\right)$

where $t_{\mathrm{p}}$ is the average travel time of the particles through the column. Here $k$ represents a time- and distance-averaged parameter. The term $C_{\mathrm{f}} / C_{0}$ corresponds to the fraction of particles recovered at the column outlet after the breakthrough curve has reached a plateau. The maximum travel distance, defined by the distance at which $99.9 \%$ of the particles have been removed from the solution, can be expressed by

$L_{\max }=-\frac{v_{\mathrm{p}}}{k} \ln \left(\frac{C_{\mathrm{f}}}{C_{0}}\right)$

where $C_{\mathrm{f}} / C_{0}=0.001$.

The attachment efficiency $(\alpha)$ for particle deposition reflects the chemical interactions between particles and matrix surfaces and is, as a first approximation, independent of physical parameters such as column packing, grain size, or bulk density. Since current theories are inadequate to predict the attachment efficiency, it is common to use column experiments to determine the attachment efficiency for a given set of physicochemical conditions (i.e., suspended particles, porous medium, and solution chemistry) (Elimelech and O’Mellia, 1990; Tufenkji and Elimelech, 2004):

$\alpha=-\frac{2}{3} \frac{d_{\mathrm{c}}}{(1-f) L \eta_{\mathrm{T}}} \ln \left(\frac{C_{\mathrm{f}}}{C_{0}}\right)$

where $d_{\mathrm{c}}$ is the diameter of the spherical collector, $f$ is the porosity of the porous medium and $\eta_{\mathrm{T}}$ is the single-collector contact efficiency.

\section{Experiment procedures}

Twelve surface $(0-20 \mathrm{~cm})$ soils were collected from 12 Chinese provinces. The soils were air-dried, ground and passed through a $1 \mathrm{~mm}$ sieve prior to experimental use. Soil organic matter (SOM) was determined using the Walkley-Black procedure (Nelson and Sommers, 1982). Soil pH was measured at soil to deionized distilled water (DDW) ratio of 1:5 (w/v) using an Orion (Model 250A+) pH meter. Cation-exchange capacity (CEC) of the soils with soil $\mathrm{pH}<7$ was determined by the $\mathrm{BaCl}_{2}$ method while soils with soil $\mathrm{pH}>7$ were determined by the $\mathrm{CH}_{3} \mathrm{COONa}$ method (Rhoades, 1982). Zeta potential of soils in the absence or presence of $\mathrm{TiO}_{2}$ was measured using a ZetaSizer Nano Series Instrument (Malvern Instrument Ltd. UK). Soil texture (sand, silt and clay contents) was measured using a pipette method (Konert and Vandenberghe, 1997). The soil suspensions used in this study were obtained at a soil to DDW ratio of $1: 20$. Soil suspension $\mathrm{pH}$ was measured using an Orion (Model $250 \mathrm{~A}+$ ) pH meter. Dissolved organic carbon (DOC) and contents of 
magnesium and calcium in soil solutions were analyzed using a total organic carbon analyzer (Phoenix 8000, Tekmar-Dohrmann Co., USA) and inductively coupled plasma-optical emission spectroscopy (ICP-OES), respectively, after centrifuging and filtering through disposable $0.45 \mu \mathrm{m}$ membranes. Electrical conductivities of soil suspension were determined using a DDS-307 conductivity meter (Arca Technology Corporation). Ionic strength of soil solutions were calculated from the simple linear equation of ionic strength and electrical conductivity (Morrisson et al., 1990):

$I=0.0127 \times \mathrm{EC}$

where $I$ and EC are the ionic strength (M) and electrical conductivity $\left(\mathrm{mS} \mathrm{cm}^{-1}\right)$, respectively.

$\mathrm{TiO}_{2}$ nanoparticles were purchased from Gaosida Nanomaterial Company (Gilin Province, China) with an anatase phase purity of $99.9 \%$, specific surface area of $32.5 \mathrm{~m}^{2} \mathrm{~g}^{-1}$ and an average particle size of $35 \mathrm{~nm}$. $\mathrm{TiO}_{2}$ suspensions were prepared by mixing $\mathrm{TiO}_{2}$ $(500 \mathrm{mg})$ with soil $(12.5 \mathrm{~g})$ and adding DDW $(250 \mathrm{~mL})$ in Erlenmeryer flasks. $\mathrm{TiO}_{2}$ in DDW at the same ratio, with no added soil was used as control, while soil in DDW at the same ratio without $\mathrm{TiO}_{2}$ was used as a blank. The mixtures were rotated vigorously for $24 \mathrm{~h}$, transferred into $250 \mathrm{~mL}$ of beakers and allowed to settle undisturbed for up to 10 days. Throughout the settling time, aliquots of supernatant liquid $(1 \mathrm{~mL})$ were carefully periodically sampled from the top of the beaker ( $2 \mathrm{~cm}$ below surface) at each time interval and the concentration of $\mathrm{TiO}_{2}$ monitored. The aggregate size distribution of $\mathrm{TiO}_{2}$ in various soil suspensions, as well as in DDW, were determined after settling for both 0 and 10 days using Laser Particle Size Analyzer (Mastersizer 2000, Marlven, Ltd. $\mathrm{UK}$ ). At the same time, the unsettled $\mathrm{TiO}_{2}$ suspensions in DDW after 10 days were collected for injection of the resulting $\mathrm{TiO}_{2}$ suspensions into leaching experiments.

For leaching experiment, a glass column, length $20 \mathrm{~cm}$ and inner diameter of $25 \mathrm{~mm}$ was uniformly packed with $10 \mathrm{~cm}$ of air-dried soil. At the beginning of the experiment, the soil column was initially saturated with DDW from the bottom of the column gradually upward through the entire length of the column, and then the column was leached with $100 \mathrm{~mL}$ of DDW. After that the turbidity of outflow was measured, and it was found that the turbidity was $<2 \mathrm{NTU}$, suggesting that the soil particle in the outflow was negligible. Subsequently, leaching $\mathrm{TiO}_{2}$ suspensions (prepared previously) were pumped into the soil column to reach a $9 \mathrm{~cm}$ water head on the top of the column. The constant water head was maintained throughout the experiment and the effluent samples were collected at discrete leaching time intervals for the measurement of $\mathrm{TiO}_{2}$ concentrations. According to Ley et al. (1994) the soil collector diameters (i.e., the average soil particle diameters) are the sum of the sand, silt and clay particle diameters of 0.175 , 0.02 and $0.0015 \mathrm{~mm}$ multiplied by their respective percentage contents in the soil. Detailed physical parameters of the various soil columns are given in Table 1. During the course of an experiment, the initial concentration of $\mathrm{TiO}_{2}$ introduced to the column, $C_{0}$, and the concentration exiting the column, $C_{\mathrm{f}}$, was monitored to obtain breakthrough curves of $C_{\mathrm{f}} / C_{0}$ as a function of the number of pore volumes passing through the soil columns. A steady plateau value of $C_{\mathrm{f}} / C_{0}$ was then interpreted using Eqs. (4) and (6) to obtain an estimate of the deposition rate coefficient and particle attachment efficiency. $\mathrm{TiO}_{2}$ aggregation size distributions in the outflow from the various soil columns were also monitored. All experiments were conducted at room temperature $\left(20 \pm 1{ }^{\circ} \mathrm{C}\right)$.

To determine the concentration of $\mathrm{TiO}_{2}$ nanoparticles in aqueous samples, the $\mathrm{TiO}_{2}$ suspension was firstly dried in a flask by heating, followed by digestion with $5 \mathrm{~mL}$ of the sulphuric acidammonium sulphate solution (Zhang et al., 2007). The above solution was transferred quantitatively and $\mathrm{Ti}$ was determined by ICP-OES. The concentration of $\mathrm{TiO}_{2}$ in the suspension was
Table 1

Physical conditions of soil columns experienced during breakthrough experiments.

\begin{tabular}{lcllll}
\hline $\begin{array}{l}\text { Soil } \\
\text { columns }\end{array}$ & $\begin{array}{l}\text { Average soil collector } \\
\text { diameter }(\mu \mathrm{m})\end{array}$ & $\begin{array}{l}\text { Darcy velocity } \\
(\mathrm{cm} / \mathrm{h})\end{array}$ & $\begin{array}{l}\text { Péclet } \\
\text { number }\end{array}$ & $\begin{array}{l}\text { Pore volume of } \\
\text { columns } V_{0}(\mathrm{~mL})\end{array}$ & $\begin{array}{l}\text { Column } \\
\text { porosity }\end{array}$ \\
\hline BJ & 76 & 5.06 & 13.8 & 22.6 & 0.461 \\
HLJ & 120 & 1.75 & 2.64 & 27.8 & 0.567 \\
JX & 51 & 0.34 & 0.23 & 24.2 & 0.493 \\
AH & 30 & 0.41 & 0.23 & 27.8 & 0.567 \\
SX & 67 & 0.67 & 0.27 & 24.0 & 0.489 \\
CD & 74 & 1.38 & 2.64 & 28.6 & 0.583 \\
NMG & 132 & 2.71 & 4.75 & 24.2 & 0.493 \\
JS & 122 & 4.40 & 2.65 & 24.1 & 0.491 \\
CQ & 70 & 0.11 & 0.15 & 27.5 & 0.561 \\
HN & 49 & 1.06 & 2.33 & 27.6 & 0.563 \\
SD & 56 & 0.80 & 1.42 & 23.9 & 0.487 \\
LZ & 57 & 0.91 & 1.82 & 23.3 & 0.475 \\
\hline
\end{tabular}

calculated by mass balance. Statistical analyses were performed using SPSS 11.5 for Windows (SPSS Inc., USA).

\section{Results and discussion}

\subsection{Characterization of soils and soil solutions}

Selected properties of the soils and soil suspensions used in this experiment are summarized in Table 2. Soil properties varied significantly across the 12 provinces sampled. The $\mathrm{pH}$ and DOC of soil suspension varied between 6.15 and 8.58 , and 51.5 and $158.3 \mathrm{mg} \mathrm{L}^{-1}$, respectively. The ionic strength of the 12 soil suspensions ranged from 0.15 to $4.95 \mathrm{mM}$. The concentrations of magnesium and calcium ions in soil suspension were 1.18$8.59 \mathrm{mg} \mathrm{L}^{-1}$ and $0.21-72.97 \mathrm{mg} \mathrm{L}^{-1}$, respectively.

\subsection{Suspension of $\mathrm{TiO}_{2}$ nanoparticles in soil suspensions}

During the sampling of supernatant liquid ( $1 \mathrm{~mL}$ ), it was inevitable that the system became slightly disturbed. However, as much as possible, the conditions for each sampling episode were kept almost identical, and therefore the monitored $\mathrm{TiO}_{2}$ concentration in various soil suspensions should be relatively comparable if not absolutely correct. The aggregation and deposition processes of $\mathrm{TiO}_{2}$ nanoparticles were soil-dependent (Fig. 1). For BJ, HLJ, JX, AH and SX soils, with relatively low ionic strengths and high DOC contents (Table 2), $31.1-35.8 \%$ of the $\mathrm{TiO}_{2}$ particles were suspended after settling for $24 \mathrm{~h}$, which was higher than the percentage observed in DDW (25.1\%). Subsequently, the suspended $\mathrm{TiO}_{2}$ relative concentrations decreased monotonically over the initial 2-4 days, and thereafter remained almost unchanged during the following 5-10 days. On day $10,1.17-2.83 \%$ of the initial $\mathrm{TiO}_{2}$ remained in the suspension, which was equal to $23.5-56.7 \mathrm{mg} \mathrm{L}^{-1}$ $\mathrm{TiO}_{2}$. For soil suspensions of $\mathrm{LZ}, \mathrm{SD}, \mathrm{HN}, \mathrm{CD}, \mathrm{NMG}$, JS and CQ soils with relatively high ionic strengths and low DOC contents (Table 2), $\mathrm{TiO}_{2}$ nanoparticles settled from solution far more quickly and after $24 \mathrm{~h}$, more than $98 \%$ of the initial $\mathrm{TiO}_{2}$ had been deposited (inset of Fig. 1) and no $\mathrm{TiO}_{2}$ nanoparticles were detected in the suspension after settling for 5 days. $\mathrm{TiO}_{2}$ nanoparticles in two soil suspensions, those having the highest ionic strengths and lowest DOC contents (LZ and SD soil suspensions) showed the fastest deposition and no suspended $\mathrm{TiO}_{2}$ was detected after settling for $24 \mathrm{~h}$. The suspended particle size distributions of various soil suspensions, as well as for DDW, at time $=0$ and 10 days are shown in Fig. 2. In DDW, the main size distribution of $\mathrm{TiO}_{2}$ aggregates at day 0 was $0.35-7.1 \mu \mathrm{m}$. As time progressed, larger aggregates preferentially settled from solution, leaving only smaller $\mathrm{TiO}_{2}$ aggregates suspended, so that after 10 days the $\mathrm{TiO}_{2}$ aggregates suspended were only 0.04$0.35 \mu \mathrm{m}$ in size. For various soil suspensions containing $\mathrm{TiO}_{2}$ at day 


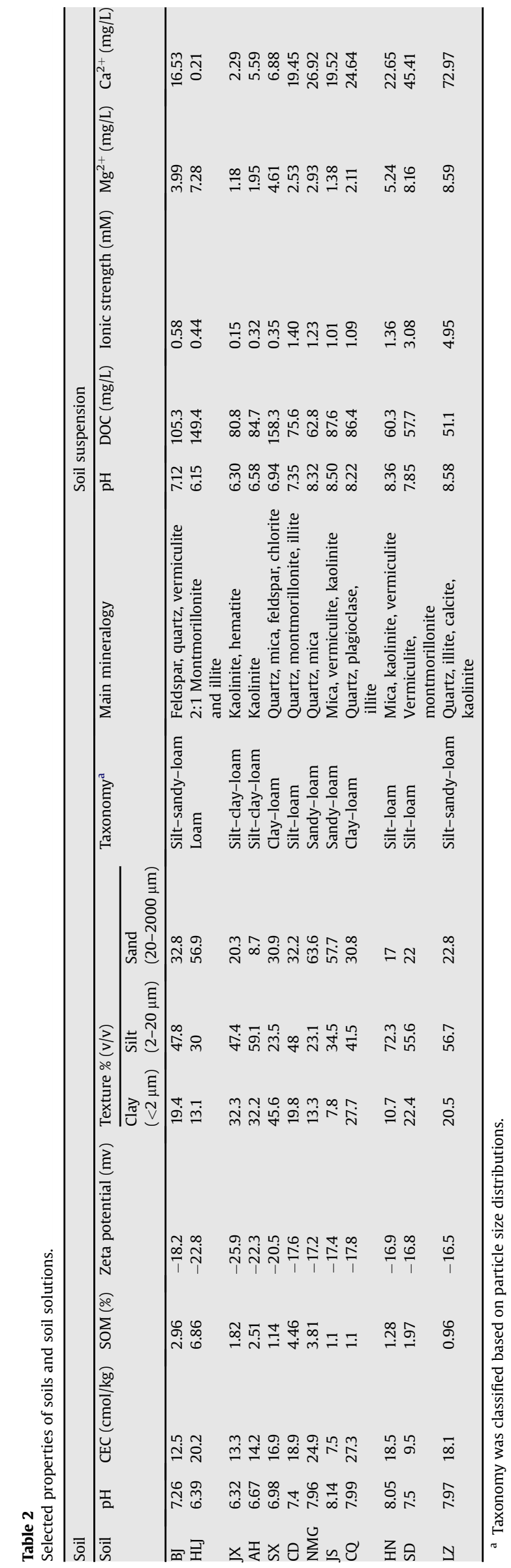

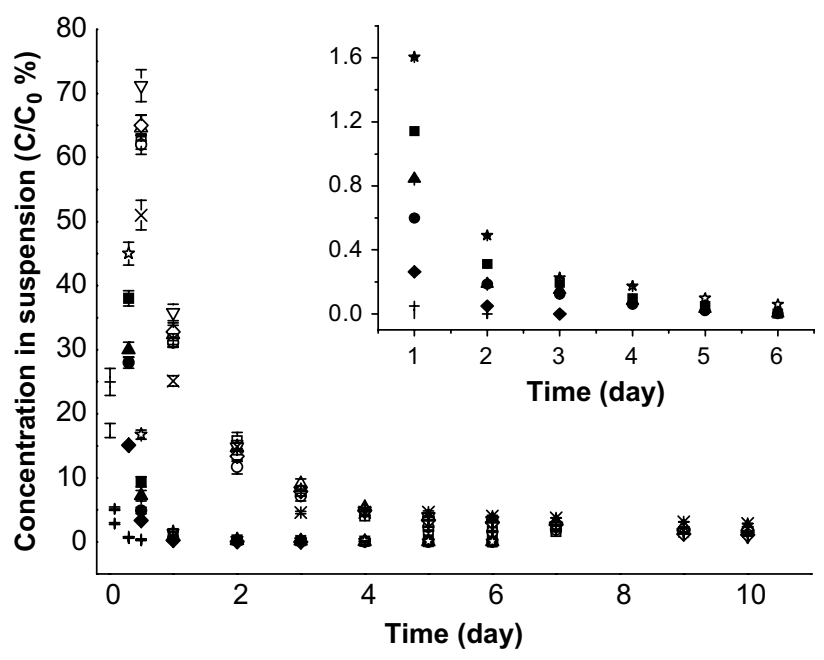

Fig. 1. Sedimentation curves of $\mathrm{TiO}_{2}$ nanoparticles in various soil suspensions. $\square$ BJ soil, $\diamond \mathrm{HLJ}$ soil, $\triangle \mathrm{JX}$ soil, $\nabla \mathrm{AH}$ soil, $\diamond \mathrm{SX}$ soil, $\square \mathrm{CD}$ soil, $\Delta \mathrm{NMG}$ soil, $\bullet \mathrm{JS}$ soil, is $\mathrm{CQ}$ soil, $\square$ HN soil, + SD soil, $\mid$ LZ soil, $\times$ DDW.

0 , the suspended particle size distribution showed much wider ranges, from 0.3 to several hundred micrometers. After settling for 10 days, milk-white suspensions of $\mathrm{TiO}_{2}$ still existed in BJ, HLJ, JX, $\mathrm{AH}, \mathrm{SX}$ soil suspensions, with very similar suspended $\mathrm{TiO}_{2}$ aggregate size distribution of $0.03-0.58 \mu \mathrm{m}$ (Fig. 2), while for the other seven soil suspensions containing $\mathrm{TiO}_{2}$, the suspensions became almost transparent (indicating almost no particles suspended) after 10 days. For the various soil suspensions without added $\mathrm{TiO}_{2}$ at day 0 , a similar wide range of particle size distributions were found which compared with those soil suspensions in the presence of $\mathrm{TiO}_{2}$. And also, most soil particles settled and the suspensions became almost transparent after 10 days (thus the suspended particle size distributions were not measured).

Principal component analysis (PCA) was performed for all 12 soils in order to define the relationships between suspension concentrations of $\mathrm{TiO}_{2}$ in soil solutions and the soil properties. The two first principle components explained $81.4 \%$ of the total variables. The principal plan plot showed two distinct soil clusters (Fig. 3a), which were explained by various factors (Fig. 3b). Soil clusters 1 (BJ, HLJ, JX, AH and SX soils) and 2 (LZ, SD, HN, CD, NMG, JS and CQ soils) were mainly grouped based on the ionic strength and DOC content. The suspended $\mathrm{TiO}_{2}$ concentrations determined after settling for $24 \mathrm{~h}$ appeared to be significantly positively correlated with DOC $(R=0.707, p<0.01)$ and clay content $(R=0.564, \quad p<0.05)$, while negatively correlated with ionic strength $(R=0.674, p<0.01), \mathrm{pH}(R=0.896, p<0.001)$ and zeta potential $(R=0.823, p<0.001)$ (Fig. 3b).

The suspensions of particles in aqueous solutions are governed by the balance of various interaction forces such as van der Waals attraction, double-layer repulsion and steric interaction (Tadros, 2007). Settling will occur when the $\mathrm{TiO}_{2}$ nanoparticles or aggregate size becomes significantly big that gravitational force overcomes buoyancy. In this work, the $\mathrm{pH}_{\mathrm{pzc}}$ of $\mathrm{TiO}_{2}$ nanoparticles was 4.5 and the zeta potential of $\mathrm{TiO}_{2}$ nanoparticles at $\mathrm{pH} \mathrm{6-8.5}$ in DDW ranged from -20.7 to $-25.8 \mathrm{mV}$. The negative surface potentials of soil particles ranged from -16.5 to $-25.9 \mathrm{mV}$ (Table 2). Thus it was expected that strong repulsion existed between negatively charged $\mathrm{TiO}_{2}$ particles and soil particles. However, based on the Derjaguin-Landau-Verwey-Overbeek (DLVO) theory, higher ionic strength compressed the diffuse double layer, causing a reduction in the repulsive electrostatic double-layer forces. Thus, higher ionic strength of cluster 2 soils (Fig. 3a) could explain the fast settling of $\mathrm{TiO}_{2}$ nanoparticles. Particles containing adsorbed or 

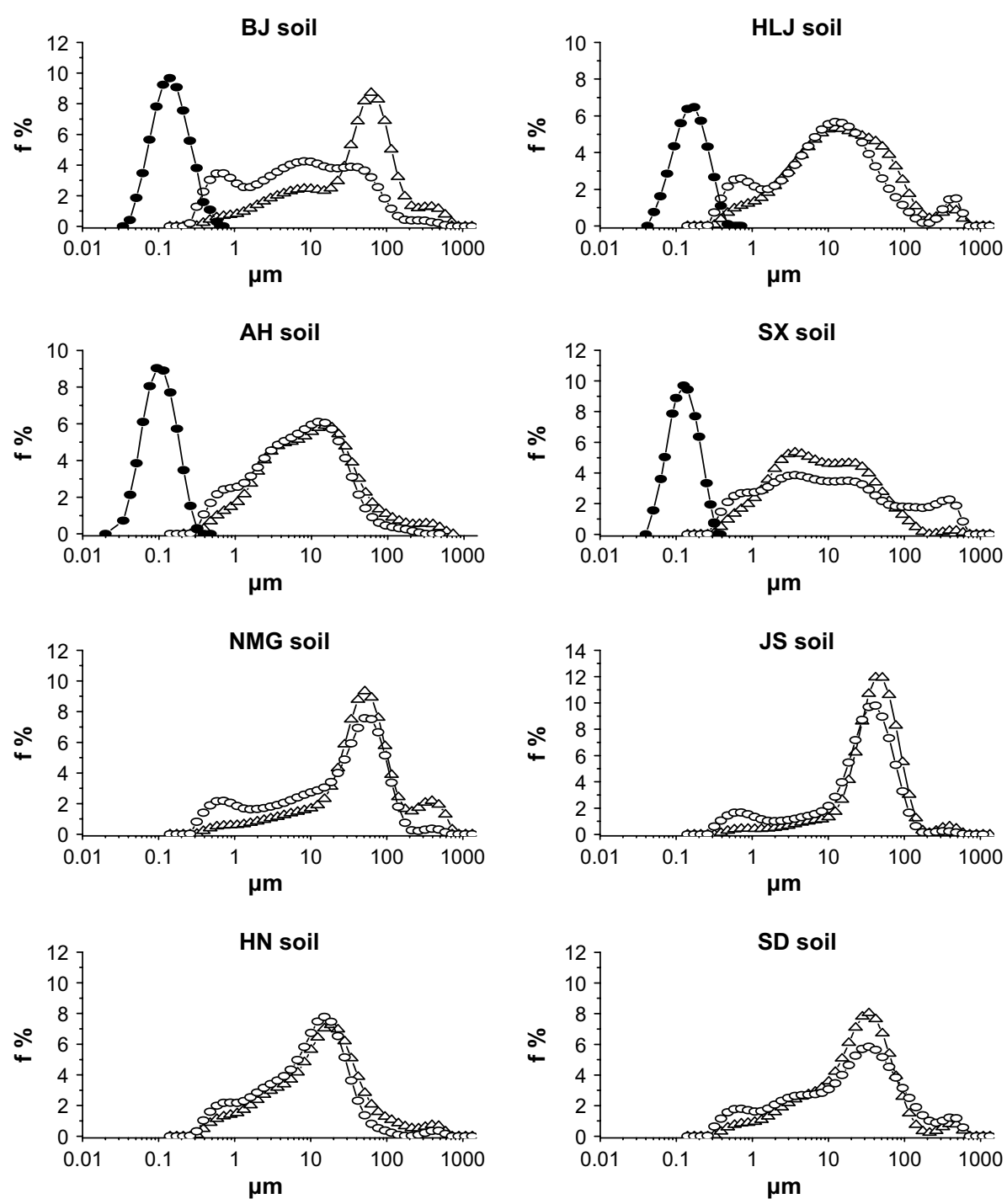
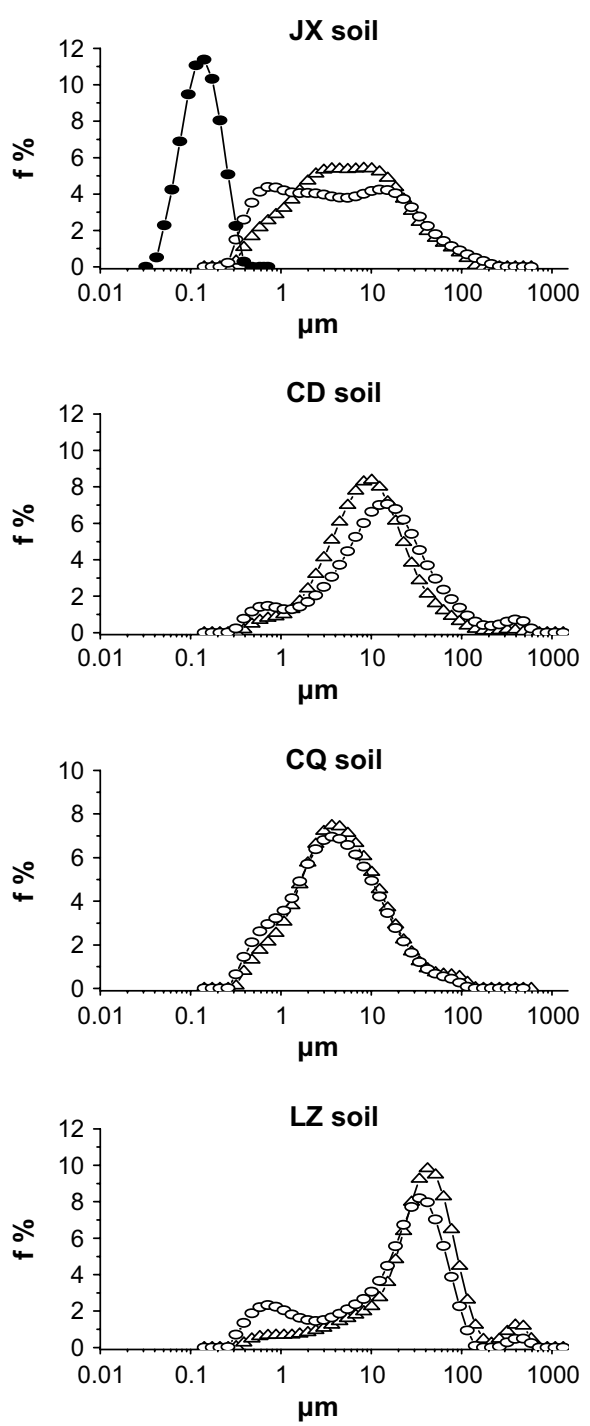

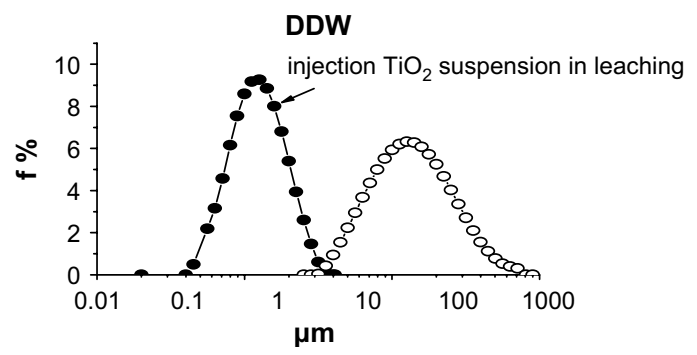

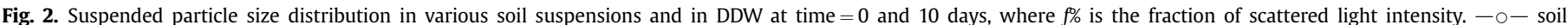

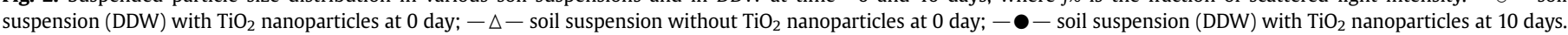

grafted surfactant or polymer layers produce another mechanism of stabilization, referred to as steric stabilization (Tadros, 2007). Since dissolved organic matter is a kind of surfactant (Hyung et al., 2007) the relatively higher DOC contents of cluster 1 soils (Fig. 3a) were expected to result in suspension of $\mathrm{TiO}_{2}$ nanoparticles. This is consistent with previous findings that DOC contributes to the stabilization of colloids in solutions (Israelachvili, 1985). Results obtained by Guzman et al. (2006) showed that when solution pH was higher than $\mathrm{pH}_{\mathrm{pzc}}$, zeta potential of $\mathrm{TiO}_{2}$ surface became more negative with increasing $\mathrm{pH}$, which resulted in increased suspension. However, in this study, the suspension $\mathrm{TiO}_{2}$ was negatively correlated to soil suspension $\mathrm{pH}$ and zeta potential (Fig. 3b), suggesting that surface potentials other than soil suspension $\mathrm{pH}$ played a key role in determining $\mathrm{TiO}_{2}$ suspension. Surface potential is an important factor influencing the magnitude of charge-based interactions of a particle, most crucially electrostatic repulsion of other like charged particles. The surface charge on a particle perturbs the ionic distribution in the medium surrounding it and $\mathrm{TiO}_{2}$ will be more stable in systems with more negative surface potential. As shown in Fig. 4, surface potentials of cluster 1 soils (below the dashed line) in the presence of $\mathrm{TiO}_{2}$ nanoparticles were much more negative than those of cluster 2 soils (above the dashed line), and also much more negative than those of their soil suspensions in the absence of $\mathrm{TiO}_{2}$ nanoparticles, and even more negative than those of $\mathrm{TiO}_{2}$ in $\mathrm{DDW}$, hence resulting in more stable suspension of $\mathrm{TiO}_{2}$ in cluster 1 soil suspensions. 

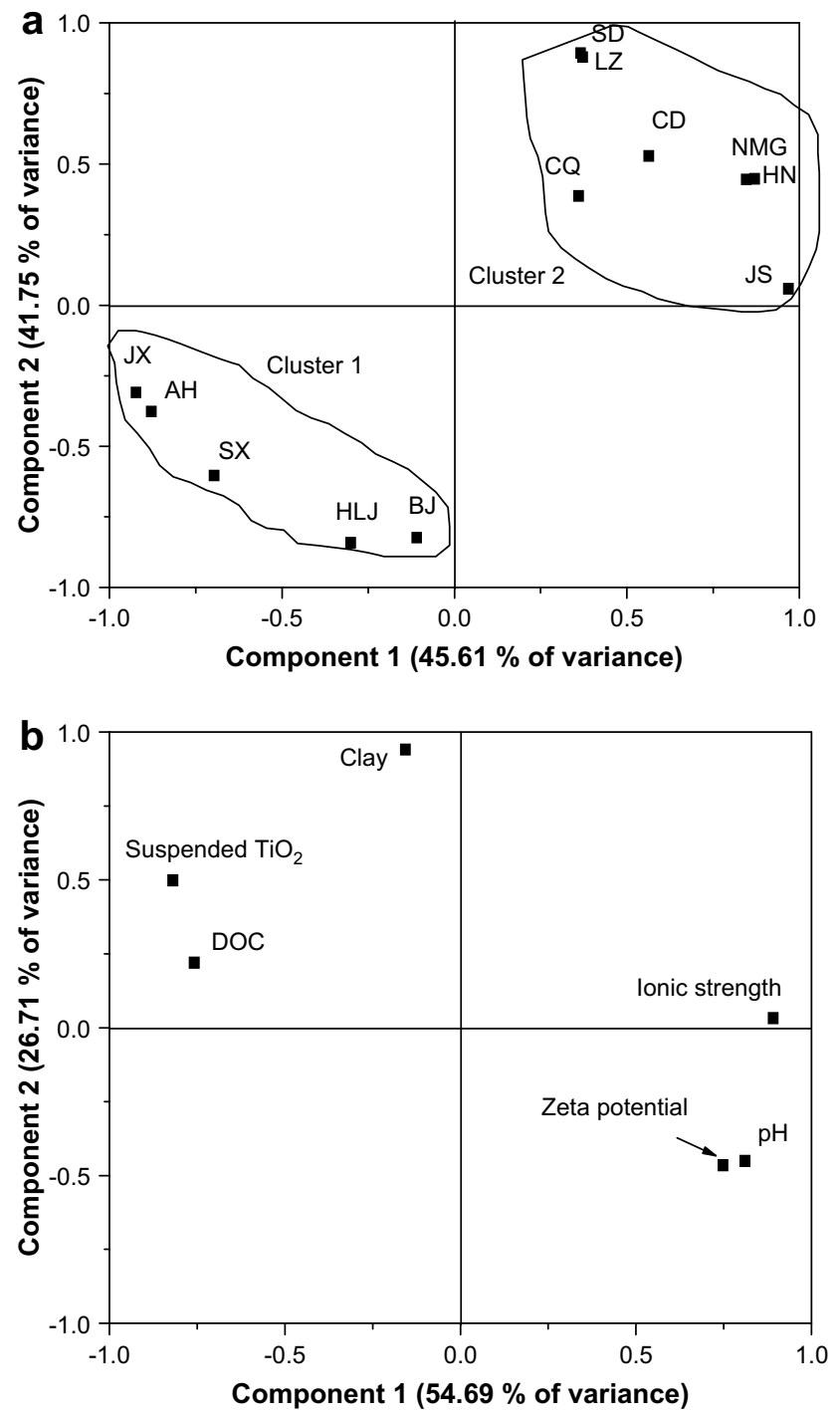

Fig. 3. Principal component analysis performed for suspension concentrations of $\mathrm{TiO}_{2}$ nanoparticles in soil suspensions and soil suspension properties. (a) Soil clusters; (b) the relationship between the unsettled $\mathrm{TiO}_{2}$ nanoparticle concentrations in soil suspensions after $24 \mathrm{~h}$ settling and soil properties.

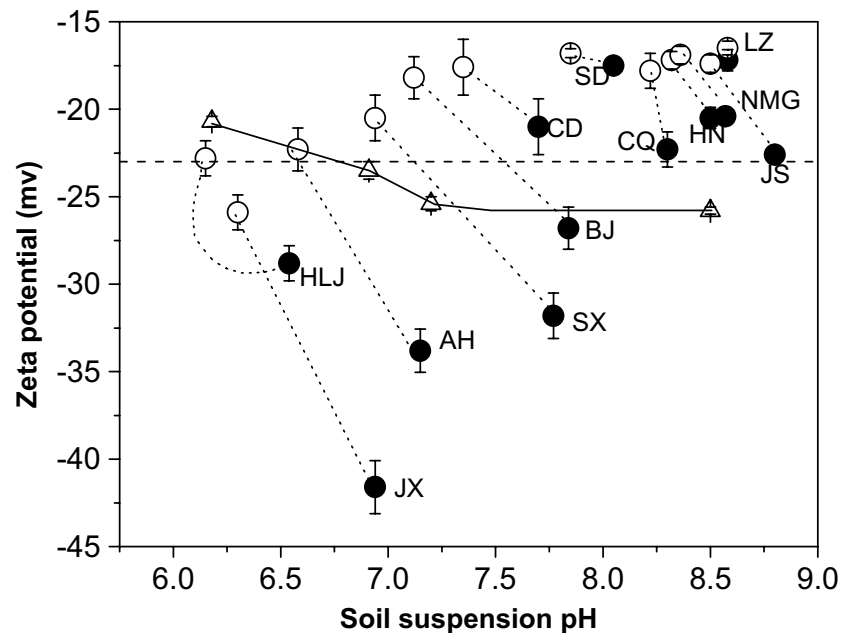

Fig. 4. Zeta potential of soil suspensions with $(\bullet)$ and without $(0) \mathrm{TiO}_{2}$ nanoparticles $\left(2 \mathrm{mg} \mathrm{L}^{-1}\right)$ as a function of soil suspension pH. $-\Delta-$ represents zeta potential of $\mathrm{TiO}_{2}$ nanoparticles in DDW.

\subsection{Transport of $\mathrm{TiO}_{2}$ nanoparticles through soil columns}

Breakthrough curves were used to indicate the mobility of $\mathrm{TiO}_{2}$ nanoparticles in various soils (Fig. 5). For soils of cluster 1 (Fig. 3a), $\mathrm{TiO}_{2}$ nanoparticles passed through BJ and HLJ soils rapidly and their existence in the first pore volume was approximately $25 \%$ and $13 \%$ of influent concentrations, respectively. Subsequently, $\mathrm{TiO}_{2}$ relative concentrations increased monotonically to the respective plateau value of $83.0 \%$ and $46.0 \%$ following the passage of $2-3$ pore volumes. While for the other soils in the same cluster, JX, AH and SX soils, more than $97 \%$ of the $\mathrm{TiO}_{2}$ nanoparticles were removed from the column flow (Fig. 5) even though they were not thought to be facilitating colloid deposition via chemical interactions. This phenomenon suggested that there may be another mechanism contributing to the suspension of $\mathrm{TiO}_{2}$ nanoparticles. Tufenkji et al. (2004) indicated that a physical removal mechanism, such as straining, which was independent of electrostatic interactions, would play an important role in particle deposition. Straining occurs within mobile-water conduits that are too narrow to permit particles to pass. Particles were retained if their diameter exceeded about one-twentieth the diameter of the porous media grains (Sakthivadivel, 1966). Indeed, straining could even be important when the ratio of the particle to median grain diameter was less than 0.002 and if the collector grains were rough and irregular in shape (Tufenkji et al., 2004; Bradford et al., 2002, 2003). Thus the heterogeneous and highly irregular soil particles contribute significantly to the porous medium straining potential. Bradford et al. (2002) and Shani et al. (2008) observed that colloid breakthrough concentrations and the final spatial distribution of colloid particles retained by porous media were highly dependent on the soil grain size distribution, especially the fine dust-like particles. Relative peak effluent concentrations decreased and surface mass removal by the soil increased when the soil median grain size decreased. The greater affinity of $\mathrm{TiO}_{2}$ to JX, $\mathrm{AH}$ and SX soil columns were due to the smaller particle diameters which resulted from high clay contents of these soils which caused a much higher average ratio of $\mathrm{TiO}_{2}$ nanoparticles to soil grain diameter and thus more straining. Harvey et al. (1993) also hypothesized that migration of clay particles was responsible for straining behavior. In addition, $\mathrm{TiO}_{2}$ nanoparticles should be highly influenced by Brownian diffusion due to their small size $(<350 \mathrm{~nm}$ ) (Guzman et al., 2006). Brownian motion will increase with decreasing size, potentially increasing the probability of nanoparticle-channel surface collisions. The extremely low Darcy velocity (Table 1) of JX, $\mathrm{AH}$ and SX soil columns may be another positive factor for $\mathrm{TiO}_{2}$ nanoparticle deposition. Lower Darcy velocity would result in more

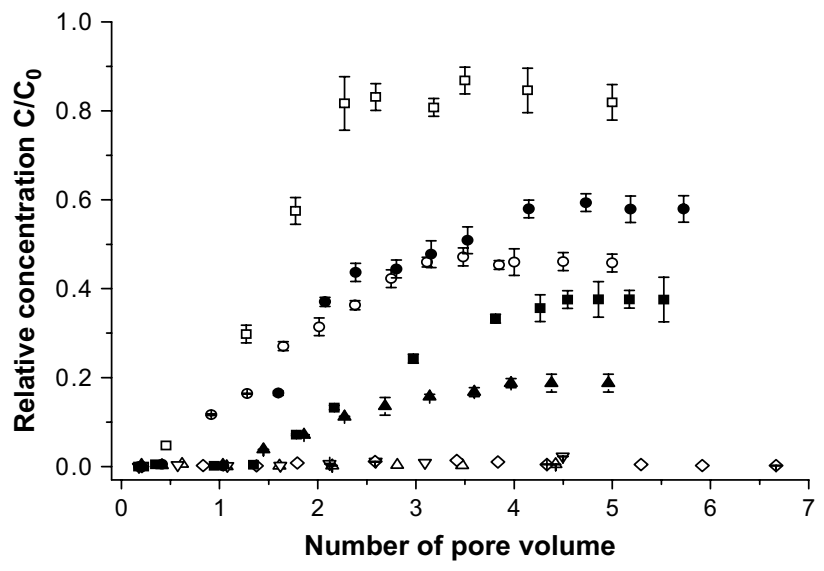

Fig. 5. Breakthrough curves of $\mathrm{TiO}_{2}$ suspensions in various soil columns. $\square$ BJ soil, HLJ soil, $\triangle$ JX soil, $\nabla$ AH soil, $\diamond$ SX soil, $\square$ CD soil, $\triangle$ NMG soil, $\bullet$ JS soil. 
efficient Brownian diffusion transport to collector surface (Lecoanet and Wiesner, 2004). Great colloid deposition occurred with decreasing flow rates (Bradford et al., 2007).

For soils in cluster 2 (Fig. 3a), the big $\mathrm{TiO}_{2}$ aggregates visually settled on the top of SD and LZ soils during transport experiments and no $\mathrm{TiO}_{2}$ breakthrough was observed. The passage of $\mathrm{TiO}_{2}$ nanoparticles was also completely blocked in CQ and HN soils and no $\mathrm{TiO}_{2}$ was detected in their effluents after leaching for 15 pore volumes. The passage of $\mathrm{TiO}_{2}$ nanoparticles in NMG, CD and JS soils tended to increase slowly with time and breakthrough started to occur after 1 pore volume and reached plateau values $\left(C_{\mathrm{f}} / C_{0}\right)$ after 4 pore volumes. The effect of ionic strength on particle deposition is in qualitative agreement with the DLVO theory of particle stability and particle deposition increased with ionic strength. Though the settling of $\mathrm{TiO}_{2}$ nanoparticles in NMG, CD and JS soil suspensions was fast (Fig. 1), significant relative concentrations of $\mathrm{TiO}_{2}$ were found in the effluent of these soils (Fig. 5). One reason responsible for the observed breakthrough could be the large soil particle diameters in these soils resulting from their high sand content. The sand grain surface roughness can influence the dynamics of particle deposition by creating shadow zones down gradient of surface protrusions where particle deposition would be significantly hindered (Ko and Elimelech, 2000).

While the size of the inflow $\mathrm{TiO}_{2}$ suspensions was between 0.03 and $0.35 \mu \mathrm{m}$ (Fig. 2-DDW), passage through the soil columns significantly changed $\mathrm{TiO}_{2}$ aggregate size (Fig. 6). After passage through the BJ, HLJ, CD, NMG and JS soil columns a new peak appeared at $1-10 \mu \mathrm{m}$ in the outflow of the soil columns, indicating that the interactions between $\mathrm{TiO}_{2}$ flow and the soil matrix favored the aggregation of $\mathrm{TiO}_{2}$ nanoparticles. The average size of the outflow $\mathrm{TiO}_{2}$ suspensions was in the order of NMG $>\mathrm{CD}>\mathrm{JS}>\mathrm{HLJ}>\mathrm{BJ}$, which was the reverse order of the $\mathrm{TiO}_{2}$ breakthrough amount for these soils, suggesting that bigger aggregates were more difficult to pass through the columns. The particle size distributions of $\mathrm{TiO}_{2}$ in the outflow of JX, AH and SX soil columns were not measured because the breakthrough concentration of $\mathrm{TiO}_{2}$ particles were not sufficient for determination.

Estimates of the particle deposition rate coefficient $(k)$ and the attachment efficiency $(\alpha)$ provide measures of a particle's mobility (Table 3). A large decrease in $\mathrm{TiO}_{2}$ nanoparticles from suspensions would suggest a higher deposition rate and collision efficiency using the first-order particle deposition model (Eq. (1)). However, experimental values of $k$ and $\alpha$ for JX, AH and SX soil columns, which had the largest decrease in $\mathrm{TiO}_{2}$ nanoparticles, were even lower than those of NMG, CD and JS soil columns (Table 3). These discrepancies might result from the ideal assumptions of the convective-diffusion model based on the classical DLVO theory and on the absence of external forces and hydrodynamic interactions (Tufenkji and Elimelech, 2004). The extremely small Péclet number for JX, AH and SX soil columns (less than 1) (Table 1) led to high single-collector contact efficiencies. The physical removal mechanisms made great contributions to the transport of particles in soil layers. In addition, nanoparticle aggregates would behave somewhat differently from individual traditional colloid particles. In this paper, $\mathrm{TiO}_{2}$ nanoparticle aggregates were considered as fractals with a lower packing density and a much higher surface area than a solid particle of similar hydrodynamic radius. Furthermore, the nanoparticle aggregates may be subject to shear forces that may break up aggregates (Guzman et al., 2006).

Another index of mobility for homogenous soil columns is the transport distance $L_{\max }$ (Table 3). Consistent with the smallest deposition rate coefficient and attachment efficiency, the longest transport distance $L_{\max }$ was obtained for soil column BJ $(370 \mathrm{~cm})$. The mobility indices $L_{\max }$ of the tested soil columns ranged from 370 to $11.1 \mathrm{~cm}$ and decreased in the order $\mathrm{BJ}>\mathrm{JS}>\mathrm{HLJ}>\mathrm{CD}>\mathrm{NMG}>\mathrm{AH}>\mathrm{SX}>\mathrm{JX}$. Thus the estimated $L_{\max }$ values were much higher than the value of $10 \mathrm{~cm}$ obtained by Lecoanet et al. (2004) for spherical glass bead columns, suggesting that $\mathrm{TiO}_{2}$ nanoparticles would transport much longer distances than previously expected due to specific soil interactions.
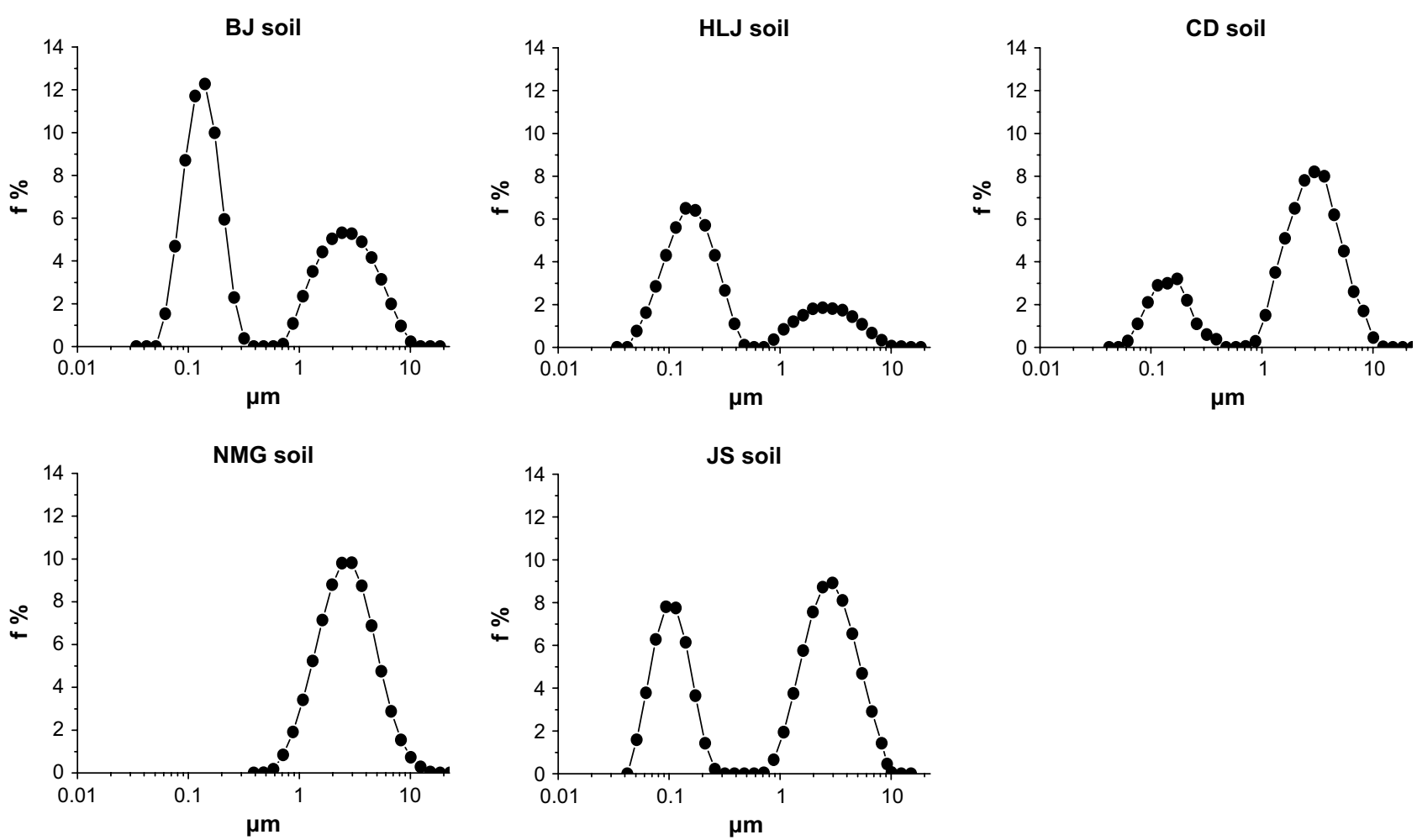

Fig. 6. Size distribution of $\mathrm{TiO}_{2}$ aggregates in solution outflow from various soil columns. 
Table 3

Transport experimental results.

\begin{tabular}{llllll}
\hline Soil column & Transported $(\%)$ & $k(1 / \mathrm{h})$ & $\eta_{\mathrm{T}}$ & $\alpha$ & $L_{\max }(\mathrm{cm})$ \\
\hline BJ & 83 & 0.0946 & 2.14 & $7.93 \mathrm{E}-05$ & 370 \\
HLJ & 46 & 0.1237 & 5.32 & $2.63 \mathrm{E}-04$ & 97.8 \\
JX & 0.2 & 0.2115 & 30 & $1.37 \mathrm{E}-04$ & 11.1 \\
AH & 2.3 & 0.1539 & 26.7 & $6.48 \mathrm{E}-05$ & 18.3 \\
SX & 1.3 & 0.2944 & 27.2 & $1.40 \mathrm{E}-04$ & 15.9 \\
CD & 37.6 & 0.1357 & 1.67 & $6.98 \mathrm{E}-04$ & 70.6 \\
NMG & 18.8 & 0.4537 & 4.01 & $7.22 \mathrm{E}-04$ & 41.3 \\
JS & 58.4 & 0.2367 & 5.96 & $1.44 \mathrm{E}-04$ & 128 \\
CQ & 0 & - & - & - & - \\
HN & 0 & - & - & - & - \\
SD & 0 & - & - & - & - \\
LZ & 0 & - & - & - & - \\
\hline
\end{tabular}

\section{Conclusions}

The stabilizing propensity of nanoparticles in soil solutions is an important subject for environmental scientists to consider when assessing the risk nanomaterials pose in soil systems. The present study demonstrated that the $\mathrm{TiO}_{2}$ nanoparticles are stable in soil suspensions. The suspended $\mathrm{TiO}_{2}$ relative concentrations were positively correlated with DOC and clay contents, and negatively correlated with ionic strength, zeta potential and $\mathrm{pH}$. The higher stability of $\mathrm{TiO}_{2}$ suspensions resulted in a higher mobility of $\mathrm{TiO}_{2}$ through soil layers. The calculated maximum travel distances $L_{\max }$ of BJ, HLJ, CD, NMG and JS soils exceeded $30 \mathrm{~cm}$, the typical surface soil depth, indicating the severe risks to deeper soil layers or even groundwater would potentially occur in these soils. Even though some soils (CD, NMG and JS) exhibited conditions that did not facilitate suspension of $\mathrm{TiO}_{2}$ nanoparticles, very small aggregates of $\mathrm{TiO}_{2}$ could still be mobilized to quite deep distances. Since, in natural systems, the wide existence of macropores formed by soil fauna, plant roots, cracks and fissures are all well known to enhance the flow of water, solutes, microorganisms, and particulate matter (Beven and Germann, 1982). Future work should study the transport of $\mathrm{TiO}_{2}$ in field soil columns, in order to better understand the fate and behavior of these nanoparticles in natural systems.

\section{Acknowledgments}

This work was funded by the National Natural Science Foundation of China (grant numbers: 20707037 and 40730740).

\section{References}

Aarthi, T., Madras, G., 2007. Photocatalytic degradation of Rhodamine dyes with nano- $\mathrm{TiO}_{2}$. Industrial and Engineering Chemistry Research 46, 7-14.

Barringer, E.A., Bowen, H.K., 1985. High-purity, monodisperse $\mathrm{TiO}_{2}$ powders by hydrolysis of titanium tetraethhoxide. 2. Aqueous interfacial electrochemistry and dispersion stability. Langmuir 1, 420-428.

Beven, K., Germann, P., 1982. Macropores and water flow in soils. Water Resources Research 18, 1311-1325.

Black, A.S., Campbell, A.S., 1982. Ionic strength of soil solution and its effect on charge properties of some New Zealand soils. European Journal of Soil Science 33, 249-262.

Bradford, S.A., Yates, S.R., Bettahar, M., Simunek, J., 2002. Physical factor affecting the fate and transport of colloid in saturated porous media. Water Resources Research 38, 1327-1338.

Bradford, S.A., Simunek, J., Bettahar, M., Van Genuchten, M., Yates, S.R., 2003. Modeling colloid attachment, straining, and exclusion in saturated porous media. Environmental Science \&Technology 37, 2242-2250.

Bradford, S.A., Torkzaban, S., Walker, S.L., 2007. Coupling of physical and chemical mechanisms of colloid straining in saturated porous media. Water Research 41, 3012-3024.

Chen, K.L., Elimelech, M., 2007. Influence of humic acid on the aggregation kinetics of fullerence $\left(C_{60}\right)$ nanoparticles in monovalent and divalent electrolyte solutions. Journal of Colloid and Interface Science 309, 126-134.

Elimelech, M., O'Mellia, C.R., 1990. Kinetics of deposition of colloidal particles in porous media. Environmental Science \&Technology 24, 1528-1536.
Guzman, K.A.D., Finnegan, M.P., Banfield, J.F., 2006. Influence of surface potential on aggregation and transport of titania nanoparticles. Environmental Science \&Technology 40, 7688-7693.

Harvey, R.W., Kinner, N.E., MacDonald, D., Metge, D.W., Bunn, A., 1993. Role of physical heterogeneity in the interpretation of small-scale laboratory and field observations of bacteria, microbial-sized microsphere, and bromide transport through aquifer sediments. Water Resources Research 29, 2713-2721.

Higarashi, M.M., Jardim, W.F., 2002. Remediation of pesticide contaminated soil using $\mathrm{TiO}_{2}$ mediated by solar light. Catalysis Today 76, 201-207.

Hsu, J., Chang, Y., 2000. An experimental study of the stability of $\mathrm{TiO}_{2}$ particles in organic-water mixtures. Colloids and Surfaces A 161, 423-437.

Hamaker, H.C., 1937. The London-van der Waals attraction between spherical particles. Physica 4 (10), 1058-1072.

Hyung, H., Fortner, J.D., Hughes, J.B., Kim, J., 2007. Natural organic matter stabilizes carbon nanotubes in the aqueous phase. Environmental Science \&Technology 41, 179-184.

Israelachvili, J.N., 1985. Intermolecular and Surface Forces With Applications to Colloidal and Biological Systems. Academic Press, New York (Chapters 11 and 12).

Kallay, N., Zalac, S., 2002. Stability of nanodispersions: a model for kinetics of aggregation of nanoparticles. Journal of Colloid and Interface Science 253, 7076.

Konert, M., Vandenberghe, J., 1997. Comparison of laser grain-size analysis with pipette and sieve analysis: a solution for the underestimation of the clay fraction. Sedimentology 44, 523-535.

Ko, C., Elimelech, M., 2000. The "shadow effect" in colloid transport and deposition dynamics in granular porous media: measurements and mechanisms. Environmental Science \&Technology 34, 3681-3689.

Kretzschmar, R., Barmettler, K., Grolimund, D., Yan, Y., Borkovec, M., Sticher, H., 1997. Experimental determination of colloid deposition rates and collision efficiencies in natural porous media. Water Resources Research 33, 1129-1137.

Lecoanet, H.F., Bottero, J., Wiesner, M.R., 2004. Laboratory assessment of the mobility of nanomaterials in porous media. Environmental Science \&Technology 38, 5164-5169.

Lecoanet, H.F., Wiesner, M.R., 2004. Velocity effects on fullerene and oxide nanoparticle deposition on porous media. Environmental Science \&Technology 38 4377-4382.

Ley, T.W., Stevens, R.G., Topielec, R.R., Neibling, W.H., 1994. Soil Water Monitoring and Measurement. Pacific Northwest Publication, Washington, Oregon, Idaho.

Mattigod, S.V., Fryxell, G.E., Alford, K., Gilmore, T., Parker, K., Serne, J., Engelhard, M., 2005. Functionalized $\mathrm{TiO}_{2}$ nanoparticles for use for in situ anion immobilization. Environmental Science \&Technology 39, 7306-7310.

Morrisson, A.R., Park, J.S., Sharp, B.L., 1990. Application of high-performance sizeexclusion liquid chromatography to the study of copper speciation in waters extracted from sewage sludge treated soils. Analyst 115, 1429-1433.

Nagaveni, K., Sivalingam, G., Hegde, M.S., Madras, G., 2004. Photocatalytic degradation of organic compounds over combustion-synthesized nano-TiO 2 . Environmental Science \&Technology 38, 1600-1604.

Nelson, D.W., Sommers, L.E., 1982. Total carbon, organic carbon, and organic matter Part 2. In: Page, A.L. (Ed.), Methods of Soil Analysis, second ed. ASA and SSSA Madison, WI, pp. 539-579.

Nowack, B., Bucheli, T.D., 2007. Occurrence, behavior and effects of nanoparticles in the environment. Environmental Pollution 150, 5-22.

Quan, X., Zhao, X., Chen, S., Zhao, H., Chen, J., Zhao, Y., 2005. Enhancement of $p, p^{\prime}$-DDT photodegradation on soil surfaces using $\mathrm{TiO}_{2}$ induced by UV-light. Chemosphere 60, 266-273.

Reilly, C., 2002. Metal Contamination of Food: Its Significance for Food Quality and Human Health, third ed. Blackwell Science, Oxford.

Part 2 Rhoades, J.D., 1982. Cation-exchange capacity. In: Page, A.L. (Ed.), Methods of Soil Analysis, second ed. America Society of Agronomy, Madison, WI, pp. 149-158.

Ryan, J.N., Elimelech, M., 1996. Colloid mobilization and transport in groundwater Colloids and Surfaces A 107, 1-56.

Sakthivadivel, T., 1966. Theory and Mechanism of Filtration of Non-colloidal Fines Through a Porous Medium; Rep HEL 15-7. Hydraulic Engineering Laboratory: University of California, Berkeley.

Sen, T.K., Khilar, K.C., 2006. Review on subsurface colloids and colloid-associated contaminant transport in saturated porous media. Advances in Colloid and Interface Science 119, 71-96.

Shani, C., Weisbrod, N., Yakirevich, A., 2008. Colloid transport through saturated sand columns: influence of physical and chemical surface properties on deposition. Colloids and Surfaces A 316, 142-150.

Snoswell, D.RE, Duan, J. Fornasiero, D. Ralston, J, 2005. Colloid stability of synthetic titania and the influence of surface roughness. Journal of Colloid and Interface Science 286, 526-535.

Tadros, T.F., 2007. Colloid Stability: the Role of Surface Forces. Part I. Wiley-VCH Verlag Gmbh \& Co. kGaA, Weinheim.

Tkachenko, N.H., Yaremko, Z.M., Bellmann, C., Soltys, M.M., 2006. The influence of ionic and nonionic surfactants on aggregative stability and electrical surface properties of aqueous suspensions of titanium dioxide. Journal of Colloid and Interface Science 299, 686-695.

Tufenkji, N., Miller, G.F., Ryan, J.N., Harvey, R.W., Elimelech, M., 2004. Transport of Cryptosporidium oocysts in porous media: role of straining and physicochemical filtration. Environmental Science \&Technology 38, 5932-5938.

Tufenkji, N., Elimelech, M., 2004. Correlation equation for predicting singlecollector efficiency in physicochemical filtration in saturated porous media. Environmental Science \&Technology 38, 529-536. 
Van Hees, P.A.W., Jones, D.L., Finlay, R., Godbold, D.L., Lundstrom, U.S., 2005. The carbon we do not see - the impact of low molecular weight compounds on carbon dynamics and respirations in forest soils: a review. Soil Biology and Biochemistry 37, 1-13.

Wiesner, M.R., Lowry, G.V., Alvarez, P., Dionysiou, D., Biswas, P., 2006. Assessing the risks of manufactured nanomaterials. Environmental Science \&Technology 40, 4336-4345.

Wilkinson, K.J., Joz-Roland, A., Buffle, J., 1997. Different roles of pedogenic fulvic acids and aquagenic biopolymers on colloid aggregation and stability in freshwaters. Limnology and Oceanography 42 (8), 1714-1724.
Yao, K.M., Habibian, M.T., O’Melia, C.R., 1971. Water and waste water filtration. Concepts and applications. Environmental Science \&Technology 5, 1105-1112.

Zhang, X., Sun, H., Zhang, Z., Niu, Q., Chen, Y., Crittenden, J.C., 2007. Enhanced bioaccumulation of cadmium in carp in the presence of titanium dioxide nanoparticles. Chemosphere 67, 160-166.

Zhuang, J., Flury, M., Jin, Y., 2003. Colloid-facilitated Cs transport through watersaturated Hanford sediment and Ottawa sand. Environmental Science \&Technology 37, 4905-4911. 